\title{
Research Article \\ Resilient Finite-Time Controller Design of a Class of Stochastic Nonlinear Systems
}

\author{
Zhiguo Yan \\ School of Electrical Engineering and Automation and Key Laboratory of Pulp and Paper Science and Technology of \\ Ministry of Education of China, Jinan 250353, China
}

Correspondence should be addressed to Zhiguo Yan; yanzg500@sina.com

Received 29 January 2014; Accepted 24 March 2014; Published 11 June 2014

Academic Editor: Hui Zhang

Copyright (C) 2014 Zhiguo Yan. This is an open access article distributed under the Creative Commons Attribution License, which permits unrestricted use, distribution, and reproduction in any medium, provided the original work is properly cited.

This paper deals with the problem of resilient finite-time control for a class of stochastic nonlinear systems. The notion of finitetime annular domain stability of stochastic nonlinear systems is first introduced. Then, some sufficient conditions are given for the existence of resilient state feedback finite-time annular domain stabilizing controller, which are expressed in terms of matrix inequalities. A double-parameter searching algorithm is proposed to solve these obtained matrix inequalities. Finally, an example is given to illustrate the effectiveness of the developed method.

\section{Introduction}

Finite-time stability is a concept that was first introduced in the 1950s, which plays an important role in the study of the transient behavior of systems. Roughly speaking, a system is said to be finite-time stable (FTS) if, given a bound on the initial condition, its state does not exceed a certain threshold during a specified time interval. Various developments and extensions in the field of FTS have been implemented, most of which have been applied to linear systems [1-4] and nonlinear systems [5-7]. Nevertheless, the FTS in [1-7] not only requires the state trajectory does not exceed a given upper bound during a prespecified time interval, but it has no requirement for the lower bound of state trajectory. Recently, [8] gave a new "finite-time stability" for linear Itô stochastic systems. In fact, this kind of stability is called "finite-time annular domain stability" (FTADS for short) more precisely. Roughly speaking, a system is FTADstable if its state trajectories do not exceed an upper bound $c_{2}$ and are not less than a lower bound $c_{1}\left(c_{1}<c_{2}\right)$ during the specific time interval. The FTADS can be used to solve some problems not only from engineering practice, such as chemical reaction temperature controlled systems and electronic circuit systems [8], but also from medicine. For example, the body's normal systolic blood pressure is 90
$130 \mathrm{mmHg}$. If the body's systolic blood pressure is less than $90 \mathrm{mmHg}$, then one suffers from low blood pressure disease [9].

On the other hand, stochastic nonlinear systems have attracted considerable attention and have become a popular research field of modern control theory [1013]. Reference [10] investigates $H_{\infty}$ control problem for a class of stochastic nonlinear systems with both state and disturbance-dependent noise. References [11, 12] studied the finite/infinite horizon mixed $\mathrm{H}_{2} / \mathrm{H}_{\infty}$ control problem for the stochastic nonlinear systems with $(x, u, v)$-dependent noise, respectively. Reference [13] addressed stochastic passivity, feedback equivalence, and global stabilization for a class of stochastic nonlinear systems.

In the implement of state feedback control, there are often some perturbations appearing in controller gain, which may result in either the actuator degradations or the requirements for readjustment of controller gains during the controller implementation stage. Therefore, it is necessary and reasonable that any controller should be able to tolerate some levels of its gain variations, which motivates us to study the resilient (nonfragile) state feedback controller problems. Although there have been some study on designing the resilient (nonfragile) controller $[14,15]$, up to date, to the 
author's knowledge, the issue of resilient finite-time control for stochastic nonlinear systems has not been investigated.

In this paper, we consider the problem of resilient FTADstabilization for a class of stochastic nonlinear systems with norm-bounded and time-varying uncertainties. By stochastic analysis technology, Gronwall inequality, and neural network method, some sufficient conditions are obtained for the existence of resilient state feedback finite-time stabilizing controller. The contributions of this paper lie in the following two aspects. (1) The concept of FTADS is extended to a class of stochastic nonlinear systems with norm-bounded and time-varying uncertainties. More precisely, a system is said to be FTAD-stable if, given a bound on the initial state of the system, the state trajectories of the system do not exceed an upper bound $c_{2}$ and are not less than a lower bound $c_{1}\left(c_{1}<c_{2}\right)$ in the mean square sense during a prespecified time interval for all admissible uncertainties. (2) The problem of resilient FTAD-stabilization is investigated and a resilient state feedback controller is designed such that the resulting closed-loop system is FTAD-stable for all admissible uncertainties.

The paper is organized as follows. In Section 2, system description along with necessary assumption is given. Section 3 provides main results. An example is analyzed to illustrate the results of the paper in Section 4. Section 5 gives the conclusion.

Notation. $A^{T}$ is transpose of a matrix or vector $A . A>0(A \geq$ $0)$ is positive definite (positive semidefinite) symmetric matrix. $L_{\mathscr{F}}^{2}\left([0, T], \mathbf{R}^{l}\right)$ is space of nonanticipative stochastic process $y(t) \in \mathbf{R}^{l}$ with respect to an increasing $\sigma$-algebra $\mathscr{F}_{t}(t \geq 0)$ satisfying $\mathbb{E} \int_{0}^{T}\|y(t)\|^{2} d t<\infty$. $I_{n \times n}$ is $n \times n$ identity matrix. $\operatorname{tr}(A)$ is trace of a matrix $A . \lambda_{\max }(A)\left(\lambda_{\min }(A)\right)$ is the maximum (minimum) eigenvalue of a real matrix $A$. $\mathbb{E}\{\cdot\}$ stands for the mathematical expectation operator with respect to the given probability measure $P$. The asterisk “*” in a matrix is used to represent the term which is induced by symmetry.

\section{Preliminaries and Problem Statement}

Consider the following stochastic nonlinear system:

$$
\begin{gathered}
d x(t)=\left[\left(A_{1}+\Delta A_{1}(t)\right) x(t)+B_{1} u(t)+f(x(t))\right] d t \\
+\left[\left(A_{2}+\Delta A_{2}(t)\right) x(t)+B_{2} u(t)\right] d w(t), \\
f(0)=0, \quad x(0)=x_{0} \in \mathbf{R}^{n},
\end{gathered}
$$

where $x(t) \in \mathbf{R}^{n}, u(t) \in L_{\mathscr{F}}^{2}\left(\mathbf{R}_{+}, \mathbf{R}^{m}\right)$ are called the system state, control input, respectively. $x_{0}$ is the initial state. Without loss of generality, throughout this paper, we assume $w(t)$ to be one-dimensional standard Wiener process defined on the probability space $\left(\Omega, \mathscr{F}_{t}, \mathscr{F}_{t}, P\right)$ with $\mathscr{F}_{t}=\sigma\{w(s)$ : $0 \leq s \leq t\} \cdot f(x(t))$ is assumed to be Borel measurable functions of suitable dimensions such that (1) has a unique strong solution on any finite interval $[0, T]$; see [16]. $A_{1}, A_{2} \in$ $\mathbf{R}^{n \times n}, B_{1}, B_{2} \in \mathbf{R}^{n \times m}$ are constant matrices. $\Delta A_{1}(t), \Delta A_{2}(t)$ are unknown matrices with time-varying uncertainties and satisfy the following conditions:

$$
\left[\begin{array}{ll}
\Delta A_{1} & \Delta A_{2}
\end{array}\right]=\operatorname{MF}(t)\left[\begin{array}{ll}
N_{1} & N_{2}
\end{array}\right],
$$

where $M, N_{1}$, and $N_{2}$ are known matrices with appropriate dimensions; $F(t): \mathbf{R} \rightarrow \mathbf{R}^{k \times l}$ is an unknown time-varying matrix function, which satisfies

$$
F^{T}(t) F(t) \leq I, \quad \forall t>0 .
$$

The parameter uncertainties are said to be admissible if (2) and (3) hold.

Remark 1. This kind of model (1) contains a large class of practical systems and has been widely investigated in control, filtering, and stability analysis [17-20].

Next, using LDI technique mentioned, nonlinear function $f(x(t))$ is to be parameterized by multilayer neural networks (MNNs). Here, we use the method in [21-23]. For the readers' convenience, the concrete process is as follows. Let the single hidden layer perceptron $\mathcal{N}\left(x(t), \mathscr{W}_{1}, \mathscr{W}_{2}\right)$ be suitably trained to approximate the nonlinear term $f(x(t))$, which is described in matrix-vector notation as

$$
\mathcal{N}\left(x(t), \mathscr{W}_{1}(x(t)), \mathscr{W}_{2}(x(t))\right)=\phi_{2}\left[\mathscr{W}_{2} \phi_{1}\left[\mathscr{W}_{1} x(t)\right]\right],
$$

where $\mathscr{W}_{i} \in R^{n_{h} \times n}, i=1,2$, denote the connecting weight matrices of neurons, and $\phi_{i}(\cdot)$ denotes the activation function vector of the NNs, which is defined as

$$
\phi_{i}[\gamma]=\left[\psi_{1}\left(\mu_{1}\right), \psi_{2}\left(\mu_{2}\right), \ldots, \psi_{n}\left(\mu_{n}\right)\right]^{T}
$$

in which we let

$$
\psi_{j}\left(\mu_{j}\right)=\chi_{j}\left(\frac{1-e^{-\mu / q_{j}}}{1+e^{-\mu / q_{j}}}\right), \quad q_{j}, \chi_{j}>0, j=1,2, \ldots, n .
$$

The maximum and minimum derivatives of activation function $\psi_{j}$ are defined as follows:

$$
h_{j}\left(k, \psi_{j}\right)= \begin{cases}\min _{\gamma_{j}} \frac{\partial \psi_{j}\left(\gamma_{j}\right)}{\partial \gamma_{j}}, & k=0, \\ \max _{\gamma_{j}} \frac{\partial \psi_{j}\left(\gamma_{j}\right)}{\partial \gamma_{j}}, & k=1 .\end{cases}
$$

The activation function $\psi_{j}$ can be rewritten in the following min-max form:

$$
\psi_{j}=\eta_{j}(0) h_{j}\left(0, \psi_{j}\right)+\eta_{j}(1) h_{j}\left(1, \psi_{j}\right),
$$

where $\eta_{j}(k), k=0,1$, is a set of positive real numbers associated with $\psi_{j}$ satisfying $\eta_{j}(k)>0$ and $\eta_{j}(0)+\eta_{j}(1)=1$.

According to the approximation theorem, for a given accuracy $\rho>0$, there exist constant weight matrices $\mathscr{W}_{i}^{*}$ defined as

$$
\begin{aligned}
& \left(\mathscr{W}_{1}^{*}, \mathscr{W}_{2}^{*}\right) \\
& \quad=\arg \min _{\left(\mathscr{W}_{1}^{*}, \mathscr{W}_{2}^{*}\right)}\left\{\max _{x(t) \in D}\left\|f(x(t))-\mathcal{N}\left(x(t), \mathscr{W}_{1}, \mathscr{W}_{2}\right)\right\|\right\},
\end{aligned}
$$


where $D \in \mathbf{R}^{m}$ is a compact set, such that

$$
\max _{x(t) \in D}\left\|f(x(t))-\mathcal{N}\left(x(t), \mathscr{W}_{1}^{*}, \mathscr{W}_{2}^{*}\right)\right\| \leq \rho\|x(t)\| .
$$

Denote a set of $n_{i}$-dimensional index vectors of the $i$ th layer $(i=1,2)$ as

$$
\kappa_{n_{i}}=\kappa_{n_{i}}(\sigma)=\left\{\sigma \in \mathbf{R}^{n_{i}} \mid \sigma_{j} \in\{0,1\}, j=1, \ldots, n_{i}\right\},
$$

where $\sigma$ is used as binary indicator. The $i$ th layer with $n_{i}$ neutrons has $2^{n_{i}}$ combinations of binary indicator with $k=$ 0,1 , and the elements of index vectors for two-layer NNs have $2^{n_{2}} \times 2^{n_{1}}$ combinations in the $\Theta=\kappa_{n_{2}} \oplus \kappa_{n_{1}}$.

By using (7) and adopting the compact representation [21], the NNs (4) can be expressed as follows:

$$
\begin{aligned}
& \mathcal{N}\left(x(t), \mathscr{W}_{1}^{*}, \mathscr{W}_{2}^{*}\right) \\
& =\phi_{2}\left[\mathscr{W}_{2}^{*}\left[\begin{array}{c}
\sum_{k=0}^{1} \eta_{1,1}(k) h_{1,1}\left(k, \psi_{1,1} \times\left(\mathscr{W}_{1}^{*} x\right)_{1}\right) \\
\vdots \\
\sum_{k=0}^{1} \eta_{1, n_{1}}(k) h_{1, n_{1}}\left(k, \psi_{1, n_{1}} \times\left(\mathscr{W}_{1}^{*} x\right)_{n_{1}}\right)
\end{array}\right]\right] \\
& =\sum_{\sigma \in \Theta} \nu_{\sigma} A_{\sigma}\left(\sigma, \phi, \mathscr{W}^{*}\right) x(t),
\end{aligned}
$$

where

$$
\begin{gathered}
A_{\sigma}=\operatorname{diag}\left[h_{2, j}\left(\sigma_{2, j}, \psi_{2, j}\right)\right] \mathscr{W}_{2}^{*} \operatorname{diag}\left[h_{1, j}\left(\sigma_{1, j}, \psi_{1, j}\right)\right] \mathscr{W}_{1}^{*} \\
\sum_{\sigma \in \kappa_{n_{2}} \oplus \kappa_{n_{1}}} \nu_{\sigma} \\
=\sum_{k_{2, n_{2}}=0, \ldots, k_{2,1}=0} \sum_{k_{2, n_{1}}=0, \ldots, k_{1,1}=0}^{1} \eta_{2, n_{2}}\left(k_{2, n_{2}}\right) \cdots \eta_{2,1}\left(k_{2,1}\right) \\
\quad \times \eta_{1, n_{1}}\left(k_{1, n_{1}}\right) \cdots \eta_{1,1}\left(k_{1,1}\right)=1 \\
\quad \eta_{i, j}\left(\sigma_{i, j}\right) \geq 0, \quad \sigma_{i j}=0,1, \\
\eta_{i, j}(0)+\eta_{i, j}(1)=1, \quad i=1,2, \quad j=n_{1}, n_{2} .
\end{gathered}
$$

Thus, by means of NNs, the resulting system (1) is transformed into a group of LDIs with error bound; that is,

$$
\begin{aligned}
d x(t)= & {\left[\left(A_{1}+\Delta A_{1}(t)+\sum_{\sigma \in \Theta} v_{\sigma} A_{\sigma}\right) x(t)\right.} \\
& \left.+B_{1} u(t)+\Delta f(x(t))\right] d t \\
+ & {\left[\left(A_{2}+\Delta A_{2}(t)\right) x(t)+B_{2} u(t)\right] d w(t), } \\
& f(0)=0, \quad x(0)=x_{0} \in \mathbf{R}^{n},
\end{aligned}
$$

where

$$
\begin{aligned}
\Delta f(x(t)) & =\max _{x(t) \in D}\left\|f(x(t))-\mathcal{N}\left(x(t), \mathscr{W}_{1}^{*}, \mathscr{W}_{2}^{*}\right)\right\| \\
& \leq \rho\|x(t)\|
\end{aligned}
$$

denotes the approximation errors of the NNs.

Remark 2. Such parameterization makes sense because any continuous nonlinear function can be approximated arbitrarily well on a compact interval by NNs.

In the following, we will extend FTADS in [8] to stochastic nonlinear systems. It is formalized through the following definition.

Definition 3. Given positive real scalars $c_{1}, c_{2}, c_{3}, c_{4}$, and $T$, with $0<c_{1}<c_{3}<c_{4}<c_{2}$, and a positive definite matrix $R$. Stochastic nonlinear system (1) with $u(t)=0$ is said to be FTAD-stable with respect to $\left(c_{1}, c_{2}, c_{3}, c_{4}, T, R\right)$ for all admissible uncertainties, if

$$
\begin{array}{r}
c_{3} \leq \mathbb{E}\left[x_{0}^{T} R x_{0}\right] \leq c_{4} \Longrightarrow c_{1}<\mathbb{E}\left[x^{T}(t) R x(t)\right]<c_{2}, \\
\forall t \in[0, T] .
\end{array}
$$

Remark 4. The FTADS requires the state trajectory not only not to exceed a given upper bound, but also not to be less than a given lower bound, which is different from FTS in [17]. The FTS only requires the state trajectory not to exceed a given upper bound. It is noted that a system which is FTS may not be FTADS. This point can be verified as follows. Although a system is FTS, its state trajectory may cross the region $\left\{x(t) \mid \mathbb{E}\left[x^{T}(t) R x(t)\right]<c_{1}\right\}$.

Next, we construct the following resilient state feedback controller for system (1):

$$
u(t)=K(t) x(t)
$$

where $K(t)=K+\Delta K(t)$ and $K$ is a constant and $\Delta K(t)$ is a perturbed matrix which is assumed to be

$$
\Delta K(t)=D_{3} F(t) N_{3},
$$

where $D_{3}$ and $N_{3}$ are known real constant matrices with appropriate dimensions and the time-varying uncertain matrix $F(t)$ satisfies (3).

Remark 5. The uncertainty part of the resilient controller (18) is supposed to be 2-norm-bounded which is fit for general parameter perturbation case.

The aim of this paper is to design resilient controller (18) such that the following closed-loop system,

$$
\begin{gathered}
d x(t)=\left[\overline{\overline{A_{1}}} x(t)+\Delta f(x(t))\right] d t+\overline{A_{2}} x(t) d w(t), \\
f(0)=0, \quad x(0)=x_{0} \in \mathbf{R}^{n}
\end{gathered}
$$

is FTAD-stable with respect to $\left(c_{1}, c_{2}, c_{3}, c_{4}, T, R\right)$, where $\overline{\overline{A_{1}}}=$ $\overline{A_{1}}+\Delta \overline{A_{1}}, \overline{A_{1}}=\sum_{\sigma \in \Theta} \nu_{\sigma} A_{\sigma}+A_{1}+B_{1} K, \Delta \overline{A_{1}}=\Delta A_{1}+B_{1} \Delta K$, 
$\overline{A_{2}}=A_{2}+B_{2} K+\Delta A_{2}+B_{2} \Delta K$, and $\bar{C}_{1}=C_{1}+D_{1} K+D_{1} \Delta K$ and

$$
\begin{aligned}
\Delta f(x(t)) & =\max _{x(t) \in D}\left\|f(x(t))-\mathcal{N}\left(x(t), \mathscr{W}_{1}^{*}, \mathscr{W}_{2}^{*}\right)\right\| \\
& \leq \rho\|x(t)\|
\end{aligned}
$$

denotes the approximation errors of the NNs.

In the following, we give some lemmas which will be used in the next sections.

Lemma 6 (Itô-type formula). For a given $V(x) \in C^{2}\left(R^{n}\right)$, associated with the following stochastic system:

$$
d x(t)=f(x) d t+g(x) d w(t),
$$

the infinitesimal generator operator is defined by

$$
\mathscr{L} V(x)=\frac{\partial V}{\partial x} f(x)+\frac{1}{2} \operatorname{Tr}\left[g^{T}(x) \frac{\partial^{2} V}{\partial x^{2}} g(x)\right] .
$$

Lemma 7 (Gronwall inequality). Let $\theta(t)$ be a nonnegative function such that

$$
\theta(t) \leq a+b \int_{0}^{t} \theta(s) d s, \quad 0 \leq t \leq T
$$

for some constants $a, b \geq 0$, and then one has

$$
\theta(t) \leq a \exp (b t), \quad 0 \leq t \leq T .
$$

Lemma 8 (see [8]). Let $\theta(t)$ be a nonnegative function such that

$$
\theta(t) \geq a+b \int_{0}^{t} \theta(s) d s, \quad 0 \leq t \leq T
$$

for some constants $a, b \geq 0$, and then one has

$$
\theta(t) \geq a \exp (b t), \quad 0 \leq t \leq T .
$$

Lemma 9. Let $L, M, F$, and $N$ be real matrices of appropriate dimension with $F^{T}(t) F(t) \leq I$. Then, for a positive scalar $\epsilon>0$, one has

$$
\begin{aligned}
L+ & M F(t) N+N^{T} F^{T}(t) M^{T} \\
& \leq L+\epsilon M M^{T}+\epsilon^{-1} N^{T} N .
\end{aligned}
$$

\section{Resilient Finite-Time Controller Design}

In this section, we consider resilient FTAD-stabilization for system (1). First, an important lemma is given.

Lemma 10. If there exist $\alpha \geq 0, \beta \geq 0$, a symmetric positive definite $Q$, and a matrix $K$ such that

$$
\begin{gathered}
{\left[\begin{array}{ll}
\amalg & \widetilde{Q} \overline{A_{2}^{T}} \\
* & -\widetilde{Q}
\end{array}\right]<0,} \\
{\left[\begin{array}{cc}
\bigsqcup & \widetilde{Q} \overline{A_{2}^{T}} \\
* & -\widetilde{Q}
\end{array}\right]<0,} \\
\frac{c_{1}}{\lambda_{\min }(Q)}<\frac{c_{2}}{\lambda_{\max }(Q)} e^{-\alpha T}, \\
c_{1} \lambda_{\max }(Q)-c_{3} \lambda_{\min }(Q)<0,
\end{gathered}
$$

then system (20) is FTAD-stable with respect to $\left(c_{1}, c_{2}, c_{3}, c_{4}, T, R\right)$, where $\widetilde{Q}=R^{-1 / 2} Q R^{-1 / 2}$, Ш= $\widetilde{\mathrm{Q}}{\overline{\overline{A_{1}^{*}}}}^{T}+\overline{\overline{A_{1}^{*}}} \widetilde{\mathrm{Q}}+2 \rho \widetilde{\mathrm{Q}}-\alpha \widetilde{\mathrm{Q}}, \sqcup=\beta \widetilde{\mathrm{Q}}-\widetilde{\overline{\mathrm{Q}}_{1}^{*}} \overline{\bar{A}}^{T}-\overline{\overline{A_{1}^{*}}} \widetilde{\mathrm{Q}}$, and $\overline{\overline{A_{1}^{*}}}=A_{\sigma}+A_{1}+B_{1} K+\Delta A_{1}+B_{1} \Delta K$.

Proof. Step 1. $\mathbb{E}\left[x^{T}(0) R x(0)\right]<c_{4} \Rightarrow \mathbb{E}\left[x^{T}(t) R x(t)\right]<c_{2}$.

Take a quadratic function $V(x(t))=x^{T}(t) \widetilde{Q}^{-1} x(t)$, where $\widetilde{Q}=R^{-1 / 2} Q R^{-1 / 2}$ with $Q>0$ being a solution to (29)-(32). Applying Itô formula for $V(x(t))$ along the trajectory of the system (20) and considering $\Delta f(x(t)) \leq \rho\|x(t)\|, \sum_{\sigma \in \Theta} \nu_{\sigma}=$ 1 , we obtain

$$
\begin{aligned}
\mathscr{L} V(x(t))= & {\left[\overline{\overline{A_{1}}} x(t)+\Delta f(x(t))\right]^{T} \widetilde{\mathrm{Q}}^{-1} x(t) } \\
& +x^{T}(t) \widetilde{\mathrm{Q}}^{-1}\left[\overline{\overline{A_{1}}} x(t)+\Delta f(x(t))\right] \\
& +x^{T}(t) \overline{A_{2}^{T}} \widetilde{\mathrm{Q}}^{-1} \overline{A_{2}} x(t) \\
\leq & x^{T}(t)\left[\overline{\overline{A_{1}^{T}}} \widetilde{\mathrm{Q}}^{-1}+\widetilde{\mathrm{Q}}^{-1} \overline{\overline{A_{1}}}+2 \rho \widetilde{\mathrm{Q}}^{-1}\right. \\
& \left.+\overline{A_{2}^{T}} \widetilde{\mathrm{Q}}^{-1} \overline{A_{2}}\right] x(t) \\
= & x^{T}(t) \sum_{\sigma \in \Theta} v_{\sigma}\left[\overline{\bar{A}_{1}^{*}} \widetilde{\mathrm{Q}}^{-1}+\widetilde{\mathrm{Q}}^{-1} \overline{\overline{A_{1}^{*}}}\right. \\
& \left.+2 \rho \widetilde{\mathrm{Q}}^{-1}+\overline{A_{2}^{T}} \widetilde{\mathrm{Q}}^{-1} \overline{A_{2}}\right] x(t),
\end{aligned}
$$

where $\overline{\overline{A_{1}^{*}}}=A_{\sigma}+A_{1}+B_{1} K+\Delta A_{1}+B_{1} \Delta K$.

Before and after multiplying (29) by

$$
\left[\begin{array}{cc}
\widetilde{Q}^{-1} & 0 \\
* & \widetilde{Q}^{-1}
\end{array}\right]
$$

(29) becomes

$$
\left[\begin{array}{cc}
{\overline{\bar{A}_{1}^{*}}}^{T} \widetilde{\mathrm{Q}}^{-1}+\widetilde{\mathrm{Q}}^{-1} \overline{\overline{A_{1}^{*}}}+2 \rho \widetilde{\mathrm{Q}}^{-1}-\alpha \widetilde{\mathrm{Q}}^{-1} & \overline{A_{2}^{T}} \widetilde{\mathrm{Q}}^{-1} \\
* & -\widetilde{\mathrm{Q}}^{-1}
\end{array}\right]<0 .
$$

According to Schur complement, (35) is equivalent to the following inequality:

$$
\begin{aligned}
{\overline{\overline{A_{1}^{*}}}}^{T} \widetilde{Q}^{-1}+\widetilde{Q}^{-1} \overline{\overline{A_{1}^{*}}}+2 \rho \widetilde{Q}^{-1} \\
+\overline{A_{2}^{T}} \widetilde{Q}^{-1} \overline{A_{2}}-\alpha \widetilde{Q}^{-1}<0 .
\end{aligned}
$$

From (33) and (36), it is easy to obtain that

$$
\mathscr{L} V(x(t))<\alpha V(x(t)) .
$$

Integrating both sides of (37) from 0 to $t$ with $t \in[0, T]$ and then taking the expectation, it yields

$$
\mathbb{E} V(x(t))<\mathbb{E} V(x(0))+\alpha \int_{0}^{t} \mathbb{E} V(x(s)) d s .
$$


By Lemma 7, we obtain

$$
\mathbb{E} V(x(t))<\mathbb{E} V(x(0)) e^{\alpha t} .
$$

According to given conditions, it follows that

$$
\begin{aligned}
\mathbb{E} V(x(t)) & =\mathbb{E}\left[x^{T}(t) R^{1 / 2} Q^{-1} R^{1 / 2} x(t)\right] \\
& \geq \lambda_{\min }\left(Q^{-1}\right) \mathbb{E}\left[x^{T}(t) R x(t)\right], \\
V(x(0)) e^{\alpha t} & =\mathbb{E}\left[x^{T}(0) R^{1 / 2} Q^{-1} R^{1 / 2} x(0)\right] e^{\alpha t} \\
& \leq \lambda_{\max }\left(Q^{-1}\right) \mathbb{E}\left[x^{T}(0) R x(0)\right] e^{\alpha t} \\
& \leq \lambda_{\max }\left(Q^{-1}\right) c_{1} e^{\alpha T} .
\end{aligned}
$$

From (40), we easily obtain

$$
\mathbb{E}\left[x^{T}(t) R x(t)\right] \leq \lambda_{\max }(Q) e^{\alpha T} \frac{c_{1}}{\lambda_{\min }(Q)} .
$$

By the condition (31), it is obvious that $\mathbb{E}\left[x^{T}(t) R x(t)\right]<c_{2}$.

Step 2. $c_{3}<\mathbb{E}\left[x^{T}(0) R x(0)\right] \Rightarrow c_{1}<\mathbb{E}\left[x^{T}(t) R x(t)\right]$.

By Schur complement, (30) is equivalent to

$$
\beta \widetilde{Q}-\widetilde{Q}{\overline{\overline{A_{1}^{*}}}}^{T}-\overline{\overline{A_{1}^{*}}} \widetilde{Q}+\widetilde{Q}{\overline{\overline{A_{2}^{*}}}}^{T} \widetilde{Q}^{-1}{\overline{\overline{A_{2}^{*}}}}^{T} \widetilde{Q}<0 .
$$

Before and after multiplying (42) by $\widetilde{Q}^{-1}$, we obtain

$$
\beta \widetilde{Q}^{-1}-{\overline{\overline{A_{1}^{*}}}}^{T} \widetilde{Q}^{-1}-\widetilde{Q}^{-1} \overline{\overline{A_{1}^{*}}}+{\overline{\overline{A_{2}^{*}}}}^{T} \widetilde{Q}^{-1} \overline{\overline{A_{2}^{*}}}<0 .
$$

Consider (33), and (43) implies

$$
\mathscr{L} V(x(t))>\beta V(x(t)) .
$$

Integrating both sides of (44) from 0 to $t$ with $t \in[0, T]$ and then taking the expectation, it yields

$$
\mathbb{E} V(x(t))>\mathbb{E} V(x(0))+\beta \int_{0}^{t} \mathbb{E} V(x(s)) d s .
$$

By Lemma 8, we conclude that

$$
\mathbb{E} V(x(t))>\mathbb{E} V(x(0)) e^{\beta t} .
$$

According to the given conditions, it follows that

$$
\begin{gathered}
\mathbb{E}\left[x^{T}(0) R^{1 / 2} Q^{-1} R^{1 / 2} x(0)\right] e^{\beta t} \\
<\mathbb{E}\left[x^{T}(t) R^{1 / 2} Q^{-1} R^{1 / 2} x(t)\right] \\
<\lambda_{\max }\left(Q^{-1}\right) \mathbb{E}\left[x^{T}(t) R x(t)\right], \\
c_{3} \lambda_{\min }\left(Q^{-1}\right)<\lambda_{\min }\left(Q^{-1}\right) \mathbb{E}\left[x^{T}(0) R x(0)\right] e^{\beta t} \\
<\mathbb{E}\left[x^{T}(0) R^{1 / 2} Q^{-1} R^{1 / 2} x(0)\right] e^{\beta t} .
\end{gathered}
$$

Because of condition (32), we obtain

$$
c_{1}<\mathbb{E}\left[x^{T}(t) R x(t)\right] .
$$

From (48), it readily follows that $c_{3}<\mathbb{E}\left[x^{T}(0) R x(0)\right]$ implies that $c_{1}<\mathbb{E}\left[x^{T}(t) R x(t)\right]$.
The following theorem gives a sufficient condition for resilient FTAD-stabilization of system (1).

Theorem 11. If there exist scalars $\alpha \geq 0, \beta \geq 0$, and positive scalars $\epsilon_{i}(i=1, \ldots, 4), \lambda_{1}, \lambda_{2}$, a symmetric positive definite $Q$, and a matrix $L$ such that

$$
\begin{gathered}
{\left[\begin{array}{ccc}
\Lambda_{11} & \Lambda_{12} & 0 \\
* & \Lambda_{22} & 0 \\
* & * & \Lambda_{33}
\end{array}\right]<0,} \\
{\left[\begin{array}{ccc}
\Phi_{11} & \Lambda_{12} & 0 \\
* & \Lambda_{22} & 0 \\
* & * & \Lambda_{33}
\end{array}\right]<0,} \\
\lambda_{1} I<Q<\lambda_{2} I, \\
c_{4} \lambda_{2} e^{\alpha T}-c_{2} \lambda_{1}<0, \\
c_{1} \lambda_{2}-c_{3} \lambda_{1}<0,
\end{gathered}
$$

then system (20) is FTAD-stable with respect to $\left(c_{1}, c_{2}, c_{3}, c_{4}, T, R\right)$, where $\Lambda_{11}=\Upsilon_{11}+2 \rho \widetilde{Q}-\alpha \widetilde{Q}$,

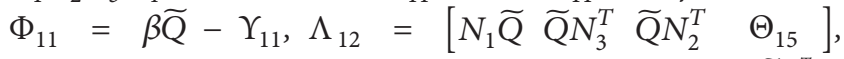

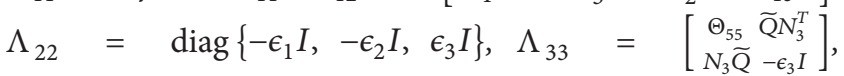
$\Theta_{15}=\widetilde{Q} A_{2}^{T}+L^{T} B_{2}^{T}, \Theta_{55}=-\widetilde{Q}+\epsilon_{3} B_{2} D_{3} D_{3}^{T} B_{2}^{T}+\epsilon_{4} M M^{T}$, $\Upsilon_{11}=\overline{\overline{A_{11}^{*}}} \widetilde{Q}+\widetilde{Q}_{\bar{A}_{11}^{*}}^{T}+B_{1} L+L^{T} B_{1}^{T}+\epsilon_{1} M M^{T}+\epsilon_{2} B_{1} D_{3} D_{3}^{T} B_{1}^{T}$, and $\overline{\overline{A_{11}^{*}}}=A_{\sigma}+A_{1}$. In this case, a desired controller gain is given by $K=L \widetilde{Q}^{-1}$.

Proof. Substitute $\overline{\overline{A_{11}^{*}}}=\overline{A_{1}^{*}}+\Delta \overline{A_{1}}, \overline{A_{1}^{*}}=A_{\sigma}+A_{1}+B_{1} K$, $\Delta \overline{A_{1}}=\Delta A_{1}+B_{1} \Delta K$, and $\overline{A_{2}}=A_{2}+B_{2} K+\Delta A_{2}+B_{2} \Delta K$ into (29) and (30), and let $\overline{\overline{A_{11}^{*}}}=A_{\sigma}+A_{1}$, (29), and (30), respectively, become

$$
\begin{gathered}
Z=\left[\begin{array}{cc}
\Xi_{11}+\Delta \Xi_{11} & \widetilde{\mathrm{Q}} A_{2}^{T}+\widetilde{\mathrm{Q}} K^{T} B_{2}^{T}+\Delta \Xi_{12} \\
* & -\widetilde{\mathrm{Q}}
\end{array}\right]<0, \\
\bar{Z}=\left[\begin{array}{cc}
\beta \widetilde{\mathrm{Q}}-\Pi+\Delta \Xi_{11} & \widetilde{\mathrm{Q}} A_{2}^{T}+\widetilde{\mathrm{Q}} K^{T} B_{2}^{T}+\Delta \Xi_{12} \\
* & -\widetilde{\mathrm{Q}}
\end{array}\right]<0,
\end{gathered}
$$

where $\Pi=\overline{\overline{A_{11}^{*}}} \widetilde{Q}+\widetilde{\bar{Q}^{*}}{ }^{T}+B_{1} K \widetilde{Q}+\widetilde{Q} K^{T} B_{1}^{T}$, $\Xi_{11}=\Pi+2 \rho \widetilde{Q}-\alpha \widetilde{Q}, \Delta \Xi_{11}=M F(t) N_{1} \widetilde{Q}+$ $\widetilde{Q} N_{1}^{T} F^{T}(t) M^{T}+B_{1} D_{3} F(t) N_{3} \widetilde{Q}+\widetilde{Q} N_{3}^{T} F^{T}(t) D_{3}^{T} B_{1}^{T}$, and $\Delta \Xi_{12}=$ $\widetilde{Q} N_{2}^{T} F^{T}(t) M^{T}+\widetilde{Q} N_{3}^{T} F^{T}(t) D_{3}^{T} B_{2}^{T}$.

In order to deal with the uncertainties described as the form in (2), we use the following approach:

$$
Z=\Xi+\Delta \Xi
$$

where

$$
\begin{gathered}
\Xi=\left[\begin{array}{cc}
\Xi_{11} & \widetilde{Q} A_{2}^{T}+\widetilde{Q} K^{T} B_{2}^{T} \\
* & -\widetilde{Q}
\end{array}\right], \\
\Delta \Xi=\left[\begin{array}{cc}
\Delta \Xi_{11} & \Delta \Xi_{12} \\
* & 0
\end{array}\right] .
\end{gathered}
$$


According to Lemma 9, we obtain the following:

$$
\begin{aligned}
\Delta \Xi & =F_{1}+F_{1}^{T}+\digamma_{2}+F_{2}^{T}+\digamma_{3}+F_{3}^{T}+F_{4}+F_{4}^{T} \\
& \left.\left.\left.\left.\left.\leq \beth_{1}+\right\lrcorner_{2}+\right\lrcorner_{3}+\right\lrcorner_{4}+\right\lrcorner_{5}+\beth_{6}+\right\lrcorner_{7}+\beth_{8} \\
& =\left[\begin{array}{cc}
\Gamma_{11} & 0 \\
* & \Gamma_{22}
\end{array}\right],
\end{aligned}
$$

where

$$
\begin{aligned}
& F_{1}=\left[\begin{array}{ll}
M^{T} & 0
\end{array}\right]^{T} F(t)\left[\begin{array}{lll}
N_{1} \widetilde{Q} & 0
\end{array}\right], \\
& F_{2}=\left[\begin{array}{ll}
D_{3}^{T} B_{1}^{T} & 0
\end{array}\right]^{T} F(t)\left[\begin{array}{ll}
N_{3} \widetilde{Q} & 0
\end{array}\right], \\
& F_{3}=\left[\begin{array}{ll}
0 & D_{3}^{T} B_{2}^{T}
\end{array}\right]^{T} F(t)\left[\begin{array}{lll}
N_{3} \widetilde{Q} & 0
\end{array}\right], \\
& F_{4}=\left[\begin{array}{ll}
0 & M^{T}
\end{array}\right]^{T} F(t)\left[\begin{array}{ll}
N_{2} \widetilde{Q} & 0
\end{array}\right], \\
& \beth_{1}=\epsilon_{1}\left[\begin{array}{ll}
M^{T} & 0
\end{array}\right]^{T}\left[\begin{array}{ll}
M^{T} & 0
\end{array}\right], \\
& \beth_{2}=\epsilon_{1}^{-1}\left[\begin{array}{ll}
\widetilde{Q} N_{1}^{T} & 0
\end{array}\right]^{T}\left[\begin{array}{ll}
\widetilde{Q} N_{1}^{T} & 0
\end{array}\right], \\
& \beth_{3}=\epsilon_{2}\left[\begin{array}{ll}
D_{3}^{T} B_{1}^{T} & 0
\end{array}\right]^{T}\left[\begin{array}{ll}
D_{3}^{T} B_{1}^{T} & 0
\end{array}\right], \\
& \beth_{4}=\epsilon_{2}^{-1}\left[\begin{array}{lll}
N_{3} \widetilde{Q} & 0
\end{array}\right]^{T}\left[\begin{array}{lll}
N_{3} \widetilde{Q} & 0
\end{array}\right], \\
& \beth_{5}=\epsilon_{3}\left[\begin{array}{ll}
0 & D_{3}^{T} B_{2}^{T}
\end{array}\right]^{T}\left[\begin{array}{ll}
0 & D_{3}^{T} B_{2}^{T}
\end{array}\right], \\
& \beth_{6}=\epsilon_{3}^{-1}\left[\begin{array}{ll}
0 & N_{3} \widetilde{Q}
\end{array}\right]^{T}\left[\begin{array}{ll}
0 & N_{3} \widetilde{Q}
\end{array}\right], \\
& \beth_{7}=\epsilon_{4}\left[\begin{array}{ll}
0 & M^{T}
\end{array}\right]^{T}\left[\begin{array}{ll}
0 & M^{T}
\end{array}\right], \\
& I_{8}=\epsilon_{4}^{-1}\left[\begin{array}{ll}
N_{2} \widetilde{Q} & 0
\end{array}\right]^{T}\left[\begin{array}{ll}
N_{2} \widetilde{Q} & 0
\end{array}\right], \\
& \Gamma_{11}=\epsilon_{1} M M^{T}+\epsilon_{1}^{-1} N_{1} \widetilde{Q} \widetilde{Q} N_{1}^{T}+\epsilon_{2} B_{1} D_{3} D_{3}^{T} B_{1}^{T} \\
& +\epsilon_{2}^{-1} \widetilde{\mathrm{Q}} N_{3}^{T} N_{3} \widetilde{\mathrm{Q}}+\epsilon_{4}^{-1} \widetilde{\mathrm{Q}} N_{2}^{T} N_{2} \widetilde{\mathrm{Q}}, \\
& \Gamma_{22}=\epsilon_{3} B_{2} D_{3} D_{3}^{T} B_{2}^{T}+\epsilon_{3}^{-1} \widetilde{Q} N_{3}^{T} N_{3} \widetilde{Q}+\epsilon_{4} M M^{T} \text {. }
\end{aligned}
$$

From the above procedure and by Schur complement,

$$
Z \leq\left[\begin{array}{ccc}
\Lambda_{11}^{*} & \Lambda_{12} & 0 \\
* & \Lambda_{22} & 0 \\
* & * & \Lambda_{33}
\end{array}\right],
$$

where $\Theta_{11}=\overline{\overline{A_{11}^{*}}} \widetilde{\mathrm{Q}}+\widetilde{\overline{\mathrm{Q}}_{11}^{*}} \overline{\mathrm{A}}^{T}+B_{1} K \widetilde{\mathrm{Q}}+\widetilde{\mathrm{Q}} K^{T} B_{1}^{T}+2 \rho \widetilde{\mathrm{Q}}-$ $\alpha \widetilde{Q}+\epsilon_{1} M M^{T}+\epsilon_{2} B_{1} D_{3} D_{3}^{T} B_{1}^{T}, \Theta_{15}=\widetilde{Q} A_{2}^{T}+\widetilde{Q} K^{T} B_{2}^{T}, \Theta_{55}=$ $-\widetilde{Q}+\epsilon_{3} B_{2} D_{3} D_{3}^{T} B_{2}^{T}+\epsilon_{4} M M^{T}, \Lambda_{11}^{*}=\Theta_{11}+2 \rho \widetilde{Q}-\alpha \widetilde{Q}, \Lambda_{12}=$ $\left[\begin{array}{lll}N_{1} \widetilde{Q} \widetilde{Q} N_{3}^{T} & \widetilde{Q} N_{2}^{T} & \Theta_{15}\end{array}\right], \Lambda_{22}=\operatorname{diag}\left\{-\epsilon_{1} I,-\epsilon_{2} I,-\epsilon_{3} I\right\}$, and $\Lambda_{33}=\left[\begin{array}{cc}\Theta_{55} & \widetilde{Q} N_{3}^{T} \\ N_{3} \widetilde{Q} & -\epsilon_{3} I\end{array}\right]$. Let $K \widetilde{Q}=L$, and the right side of (59) becomes (49), which guarantees $Z<0$. Using the same procedure, (50) guarantees $\bar{Z}<0$.

On the other hand, it is easy to check that (51) and (52) can guarantee (31), and (53) can guarantee (32).
So, according to Lemma 10, system (20) is FTAD-stable with respect to $\left(c_{1}, c_{2}, c_{3}, c_{4}, T, R\right)$.

In the special case, when $\Delta K=0$, Theorem 11 reduces the following corollary.

Corollary 12. If there exist scalars $\alpha \geq 0, \beta \geq 0$, and positive scalars $\epsilon_{1}, \epsilon_{2}, \lambda_{1}, \lambda_{2}$, a symmetric positive definite $Q$, and $a$ matrix L such that (51)-(53) and

$$
\begin{aligned}
& {\left[\begin{array}{ccc}
\Delta_{11} & \Delta_{12} & 0 \\
* & -\epsilon_{2} I & 0 \\
* & * & \Theta_{55}^{*}
\end{array}\right]<0,} \\
& {\left[\begin{array}{ccc}
\Phi_{11}^{*} & \Delta_{12} & 0 \\
* & -\epsilon_{2} I & 0 \\
* & * & \Theta_{55}^{*}
\end{array}\right]<0,}
\end{aligned}
$$

then system (20) is FTAD-stable with respect to $\left(c_{1}, c_{2}, c_{3}, c_{4}\right.$, $T, R)$, where $\Delta_{11}=\Upsilon_{11}+2 \rho \widetilde{Q}-\alpha \widetilde{Q}, \Phi_{11}^{*}=\beta \widetilde{Q}-\Upsilon_{11}, \Delta_{12}=$ $\left[N_{1} \widetilde{\mathrm{Q}} \widetilde{\mathrm{Q}} N_{2}^{T} \Theta_{15}^{*}\right], \Theta_{15}^{*}=\widetilde{\mathrm{Q}} A_{2}^{T}+L^{T} B_{2}^{T}, \Theta_{55}^{*}=-\widetilde{\mathrm{Q}}+\epsilon_{2} M M^{T}$, $\Upsilon_{11}=\overline{\overline{A_{11}^{*}}} \widetilde{Q}+\widetilde{\bar{Q}}_{\bar{A}_{11}^{*}}^{T}+B_{1} L+L^{T} B_{1}^{T}+\epsilon_{1} M M^{T}$, and $\overline{\overline{A_{11}^{*}}}=A_{\sigma}+$ $A_{1}$. In this case, a desired controller gain is given by $K=L \widetilde{Q}^{-1}$.

Remark 13. It is easy to see that the values of $\alpha$ and $\beta$ determine the feasibility of Theorem 11 and Corollary 12. The procedure how to choose $\alpha$ and $\beta$ is given in the next subsection.

Next, a double-parameter searching algorithm is given to solve the matrix inequalities in Theorem 11. Similar algorithm can be applied to Corollary 12.

Algorithm 14. Step 1. Give $c_{1}, c_{2}, c_{3}, c_{4}, T$, and $R$.

Step 2. Take a series of $\alpha_{i}(i=1, \ldots, n)$ by a step size $d_{1}$ and a series of $\beta_{j}(j=1, \ldots, m)$ by a step size $d_{2}$.

Step 3. Set $i=1$, and take a $\alpha_{i}$.

Step 4. Set $j=1$, and take a $\beta_{j}$.

Step 5. If $\left(\alpha_{i}, \beta_{j}\right)$ makes (49)-(53) have feasible solutions, then store $\left(\alpha_{i}, \beta_{j}\right)$ into $(X(i), Y(j))$ and $\beta_{j}=\beta_{j+1}$ and go to Step 5; otherwise, go to Step 6 .

Step 6. If $i+1<n$, then $\alpha_{i}=\alpha_{i+1}$ and take $\beta_{1}$ and go to Step 5. Otherwise, go to Step 7.

Step 7. Stop. If $(X, Y)=(0,0)$, then we cannot find $(\alpha, \beta)$ which makes (49)-(53) have feasible solution; otherwise, there exists $(\alpha, \beta)$ which makes (49)-(53) have feasible solution.

Remark 15. By Algorithm 14, we can obtain a region surrounded by $\alpha$ and $\beta$, if it exists, which is used to select $\alpha$ and $\beta$ for appropriate conditions.

\section{Numerical Example}

In this section, we provide an illustrative example to demonstrate the effectiveness and advantages of the proposed method. 
Example 1. Consider stochastic nonlinear system (1) with

$$
\begin{gathered}
A_{1}=\left[\begin{array}{cc}
-11 & 2 \\
1 & -15
\end{array}\right], \quad B_{1}=\left[\begin{array}{c}
-0.3 \\
0.4
\end{array}\right], \\
A_{2}=\left[\begin{array}{ll}
0.3 & 0.2 \\
0.4 & 0.5
\end{array}\right], \quad B_{2}=\left[\begin{array}{c}
0.5 \\
-0.4
\end{array}\right], \\
M=\left[\begin{array}{cc}
0.2 & 0 \\
0 & 0.3
\end{array}\right], \quad N_{1}=\left[\begin{array}{cc}
0.3 & 0 \\
0 & 0.2
\end{array}\right], \\
N_{2}=\left[\begin{array}{cc}
0.2 & 0 \\
0 & 0.15
\end{array}\right], \quad f(x(t))=\left[\begin{array}{c}
e^{x_{1}(t)} \sin x_{1}(t) \\
0
\end{array}\right] .
\end{gathered}
$$

The initial value is taken as $x(0)=[1.5-1.2]^{T}$ and the parameters $c_{1}=1, c_{2}=25, c_{3}=3, c_{4}=5, T=0.25$, and $R=I$ are given. Now, we consider a single hidden layer neural network with three hidden neurons to approximate the nonlinear function $e^{x(t)} \sin x(t)$. All parameters of activation functions (5) associated with the hidden layer are chosen to be $q_{j}=0.5, \chi_{j}=1$. For these activation functions, we have $h_{j}\left(0, \psi_{j}\right)=0, h_{j}\left(1, \psi_{j}\right)=1$. The connection weights are trained offline by using the back propagation algorithm. The initial weights and state vector are placed by uniformly distributed random numbers in $\left[\begin{array}{ll}-1 & 1\end{array}\right]$. After 1000 training steps, the optimal approximation weights are as follows:

$$
\begin{aligned}
& W_{1}^{*}=\left[\begin{array}{lll}
3.3892 & -4.4106 & -4.4786
\end{array}\right]^{T}, \\
& W_{2}^{*}=\left[\begin{array}{lll}
3.9513 & -1.4257 & -0.5506
\end{array}\right] .
\end{aligned}
$$

The upper bound of approximation error is estimated as $\rho=$ $2 \times 10^{-5}$. Obviously, in this case, we have $\Theta=2^{3} \times 2^{1}$. According to (13), $A_{\sigma}$ can be obtained as follows:

$$
\begin{aligned}
& A_{1}=A_{2}=A_{3}=A_{4}=A_{5} \\
& =A_{6}=A_{7}=A_{8}=A_{9} \\
& =A_{1 \oplus[0,0,0]^{T}}=A_{0 \oplus[i, j, k]^{T}}=\left[\begin{array}{ll}
0 & 0 \\
0 & 0
\end{array}\right], \\
& (i, j, k \in\{0,1\}) \\
& A_{10}=A_{0 \oplus[1,0,0]^{T}}=\left[\begin{array}{cc}
13.3917 & 0 \\
0 & 0
\end{array}\right], \\
& A_{11}=A_{0 \oplus[0,1,0]^{T}}=\left[\begin{array}{cc}
6.2882 & 0 \\
0 & 0
\end{array}\right], \\
& A_{12}=A_{0 \oplus[0,0,1]^{T}}=\left[\begin{array}{cc}
2.4659 & 0 \\
0 & 0
\end{array}\right],
\end{aligned}
$$

$$
\begin{gathered}
A_{13}=A_{0 \oplus[1,1,0]^{T}}=\left[\begin{array}{cc}
19.68 & 0 \\
0 & 0
\end{array}\right], \\
A_{14}=A_{0 \oplus[1,0,1]^{T}}=\left[\begin{array}{cc}
15.8576 & 0 \\
0 & 0
\end{array}\right], \\
A_{15}=A_{0 \oplus[0,1,1]^{T}}=\left[\begin{array}{cc}
8.7541 & 0 \\
0 & 0
\end{array}\right], \\
A_{16}=A_{0 \oplus[1,1,1]^{T}}=\left[\begin{array}{cc}
22.1548 & 0 \\
0 & 0
\end{array}\right] .
\end{gathered}
$$

Here, we design the following resilient state feedback controller:

$$
u(t)=\left[k_{1}+\Delta k_{1}(t) k_{2}+\Delta k_{2}(t)\right] x(t),
$$

where $k_{1}$ and $k_{2}$ will be determined and $-0.5 \leq \Delta k_{1}(t) \leq 0.5$ and $-0.5 \leq \Delta k_{2}(t) \leq 0.5$ represent some variations in the gains of the controller. Then, we have

$$
\begin{gathered}
D_{3}=\left[\begin{array}{ll}
1 & 1
\end{array}\right], \\
F(t)=\left[\begin{array}{cc}
2 \Delta k_{1}(t) & 0 \\
0 & 2 \Delta k_{2}(t)
\end{array}\right], \\
N_{3}=\left[\begin{array}{cc}
0.5 & 0 \\
0 & 0.5
\end{array}\right] .
\end{gathered}
$$

Applying Algorithm 14 to Theorem 11, a region surrounded by $\alpha$ and $\beta$ is obtained, which is illustrated by Figure 1. Selecting $\alpha=4, \beta=0$ and solving (49)-(53), we get

$$
\begin{gathered}
Q=\left[\begin{array}{ll}
0.0769 & 0.0116 \\
0.0116 & 0.1178
\end{array}\right], \quad \epsilon_{1}=0.3291, \quad \epsilon_{2}=0.3679, \\
\epsilon_{3}=0.5085, \quad \epsilon_{4}=0.2028, \quad \lambda_{1}=0.0699, \\
\lambda_{2}=0.1247, \quad L=\left[\begin{array}{ll}
0.2373 & 0.3035
\end{array}\right], \\
K=\left[\begin{array}{ll}
2.7388 & 2.3069
\end{array}\right] .
\end{gathered}
$$

Therefore, the following resilient state feedback controller,

$$
u(t)=\left[2.7388+\Delta k_{1}(t) 2.3069+\Delta k_{2}(t)\right] x(t)
$$

is obtained.

When $\Delta K=0$, a nonresilient controller will be obtained. Applying Algorithm 14 to Corollary 12, a region surrounded by $\alpha$ and $\beta$ is obtained, which is illustrated by Figure 2 . Selecting $\alpha=4, \beta=0$ and solving (51)-(53) and (60), we get

$$
\begin{gathered}
Q=\left[\begin{array}{ll}
2.9461 & 0.4550 \\
0.4550 & 4.5492
\end{array}\right], \quad \epsilon_{1}=11.9272, \\
\epsilon_{2}=13.2296, \\
\lambda_{1}=2.7195, \quad \lambda_{2}=4.8390, \\
L=\left[\begin{array}{ll}
8.5249 & 12.2147
\end{array}\right] \\
K=\left[\begin{array}{ll}
2.5178 & 2.4332
\end{array}\right] .
\end{gathered}
$$




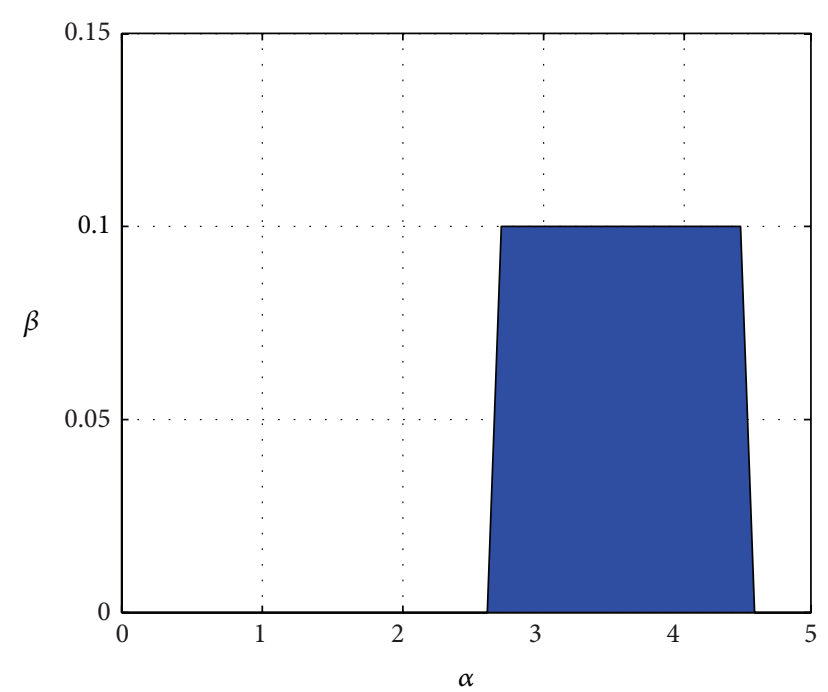

Figure 1: A region by $\alpha$ and $\beta$.

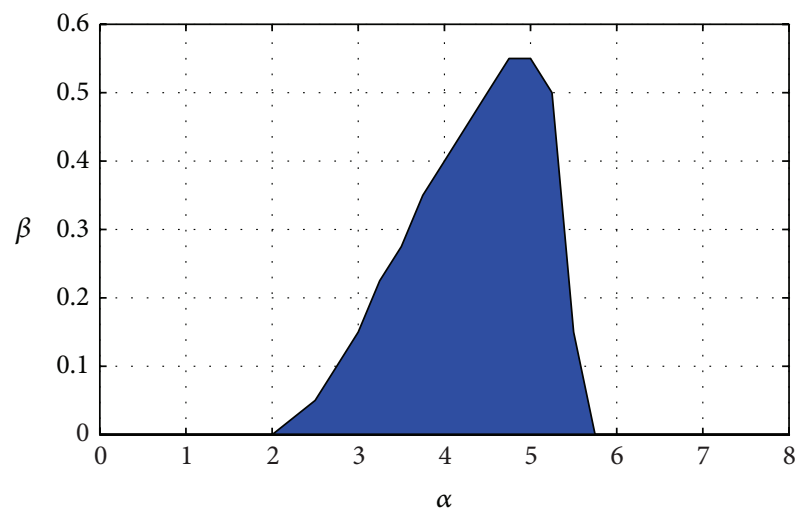

Figure 2: A region by $\alpha$ and $\beta$.

So, the following nonresilient state feedback controller,

$$
u(t)=\left[\begin{array}{ll}
2.5178 & 2.4332
\end{array}\right] x(t)
$$

is obtained.

Next, a concrete response of closed-loop system of (1) is presented in the resilient control design case. When $F(t)=I$, the parameter perturbations are specific for $\Delta A_{1}=M N_{1}, \Delta A_{2}=M N_{2}$, and $\Delta K=D_{3} N_{3}$. We, respectively, apply resilient controller (67) and nonresilient controller (69) to system (1). The evolutions of $\mathbb{E}\left[x^{T}(t) R x(t)\right]$ of closed-loop system (20) are obtained, which show that the closed-loop system of (1) is FTAD-stable with respect to $(1,3,5,25,0.25, I)$. The evolution of $\mathbb{E}\left[x^{T}(t) R x(t)\right]$ using resilient controller is lower than that using nonresilient controller in Figure 3, which shows that resilient controller is superior to nonresilient controller.

\section{Conclusion}

In this study, we have studied the problem of resilient controller design for a class of stochastic nonlinear systems.

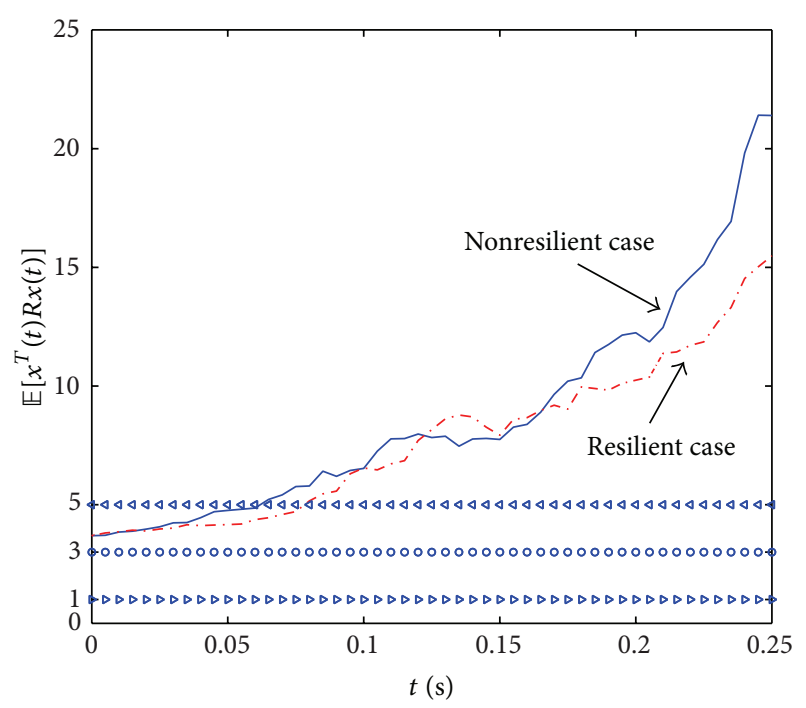

Figure 3: The response of system (1) for $\mathbb{E}\left[x^{T}(t) R x(t)\right]$.

Some sufficient conditions for the existence of resilient state feedback finite-time stabilizing controller have been obtained, which are expressed in terms of matrix inequalities. A double-parameter searching algorithm is proposed to solve these obtained matrix inequalities. One example is presented to illustrate the effectiveness of the proposed results. In addition, we can also refer to [24-27] and extend the results of this paper to networked systems, Markovian jumping systems, sampled nonlinear systems, and so on.

\section{Conflict of Interests}

The author declares that there is no conflict of interests regarding the publication of this paper.

\section{Acknowledgment}

This work is supported by the Starting Research Foundation of Qilu University of Technology (Grant no. 12045501) and Open foundation of Key Laboratory of Pulp and Paper Science and Technology of Ministry of Education of China (Grant no. 08031347).

\section{References}

[1] F. Amato, M. Ariola, and P. Dorato, "Finite-time control of linear systems subject to parametric uncertainties and disturbances," Automatica, vol. 37, no. 9, pp. 1459-1463, 2001.

[2] F. Amato, M. Ariola, and C. Cosentino, "Finite-time stabilization via dynamic output feedback," Automatica, vol. 42, no. 2, pp. 337-342, 2006.

[3] W. Zhang and X. An, "Finite-time control of linear stochastic systems," International Journal of Innovative Computing, Information and Control, vol. 4, no. 3, pp. 687-694, 2008.

[4] F. Amato, M. Ariola, and C. Cosentino, "Finite-time stability of linear time-varying systems: analysis and controller design," IEEE Transactions on Automatic Control, vol. 55, no. 4, pp. 10031008, 2010. 
[5] Y. Yang, J. Li, and G. Chen, "Finite-time stability and stabilization of nonlinear stochastic hybrid systems," Journal of Mathematical Analysis and Applications, vol. 356, no. 1, pp. 338345, 2009.

[6] Z. Yan, G. Zhang, and J. Wang, "Non-fragile robust finitetime $H_{\infty}$ control for nonlinear stochastic Itô systems using neural network," International Journal of Control, Automation and Systems, vol. 10, no. 5, pp. 873-882, 2012.

[7] Z. Yan and G. Zhang, "Finite-time $H_{\infty}$ filtering for a class of nonlinear stochastic uncertain systems," Control and Decision, vol. 29, no. 3, pp. 419-424, 2012.

[8] Z. Yan, G. Zhang, and W. Zhang, "Finite-time stability and stabilization of linear Itô stochastic systems with state and control-dependent noise," Asian Journal of Control, vol. 15, no. 1, pp. 270-281, 2013.

[9] J. Ge and Y. Xu, Internal Medicine, People's Press, 2013 (Chinese).

[10] W. Zhang and B. S. Chen, "State feedback $H_{\infty}$ control for a class of nonlinear stochastic systems," SIAM Journal on Control and Optimization, vol. 44, no. 6, pp. 1973-1991, 2006.

[11] W. Zhang, H. Zhang, and B.-S. Chen, "Stochastic $H_{2} / H_{\infty}$ control with $(x, u, v)$-dependent noise: finite horizon case," Automatica, vol. 42, no. 11, pp. 1891-1898, 2006.

[12] W. Zhang and G. Feng, "Nonlinear stochastic $H_{2} / H_{\infty}$ control with $(x, u, v)$-dependent noise: infinite horizon case," IEEE Transactions on Automatic Control, vol. 53, no. 5, pp. 1323-1328, 2008.

[13] Z. Lin, J. Liu, Y. Lin, and W. Zhang, "Nonlinear stochastic passivity, feedback equivalence and global stabilization," International Journal of Robust and Nonlinear Control, vol. 22, no. 9, pp. 999-1018, 2012.

[14] G. H. Yang and W. W. Che, "Non-fragile $H_{\infty}$ filter design for linear continuous-time systems," Automatica, vol. 44, no. 11, pp. 2849-2856, 2008.

[15] X. G. Guo and G. H. Yang, "Non-fragile $H_{\infty}$ filter design for delta operator formulated systems with circular region pole constraints: an LMI optimization approach," Acta Automatica Sinica, vol. 35, no. 9, pp. 1209-1215, 2009.

[16] X. Mao, Stochastic Differential Equations and Applications, Horwood Publishing, Chichester, UK, 2nd edition, 2008.

[17] S. Battilotti and A. De Santis, "Stabilization in probability of nonlinear stochastic systems with guaranteed region of attraction and target set," IEEE Transactions on Automatic Control, vol. 48, no. 9, pp. 1585-1599, 2003.

[18] H. Mukaidani, "The guaranteed cost control for uncertain nonlinear large-scale stochastic systems via state and static output feedback," Journal of Mathematical Analysis and Applications, vol. 359, no. 2, pp. 527-535, 2009.

[19] I. R. Petersen, "Robust output feedback guaranteed cost control of nonlinear stochastic uncertain systems via an IQC approach," IEEE Transactions on Automatic Control, vol. 54, no. 6, pp. 12991304, 2009.

[20] Z. Yan, G. Zhang, and J. Wang, "Non-fragile robust finitetime stabilization for nonlinear stochastic systems via neural network," in Proceedings of the 8th Asian Control Conference (ASCC '11), pp. 547-552, Kaohsiung, Taiwan, May 2011.

[21] S. Limanond and J. Si, "Neural-network-based control design: an LMI approach," IEEE Transactions on Neural Networks, vol. 9, no. 6, pp. 1422-1429, 1998.

[22] C. L. Lin and T. Y. Lin, "An $H_{\infty}$ design approach for neural net-based control schemes," IEEE Transactions on Automatic Control, vol. 46, no. 10, pp. 1599-1605, 2001.
[23] X. Luan, F. Liu, and P. Shi, "Robust finite-time $H_{\infty}$ control for nonlinear jump systems via neural networks," Circuits, Systems, and Signal Processing, vol. 29, no. 3, pp. 481-498, 2010.

[24] H. Zhang, Y. Shi, and A. S. Mehr, "Robust $H_{\infty}$ PID control for multivariable networked control systems with disturbance/noise attenuation," International Journal of Robust and Nonlinear Control, vol. 22, no. 2, pp. 183-204, 2012.

[25] H. Zhang, J. M. Wang, and Y. Shi, "Robust $H_{\infty}$ sliding-mode control for Markovian jump systems subject to intermittent observations and partially known transition probabilities," Systems and Control Letters, vol. 62, no. 12, pp. 1114-1124, 2013.

[26] H. Zhang, Y. Shi, and J. M. Wang, "Observer-based tracking controller design for networked predictive control systems with uncertain Markov delays," International Journal of Control, vol. 86, no. 10, pp. 1824-1836, 2013.

[27] H. Zhang, Y. Shi, and J. M. Wang, “On energy-to-peak filtering for non-uniformly sampled nonlinear systems: a Markovian jump system approach," IEEE Transactions on Fuzzy Systems, vol. 22, no. 1, pp. 212-222, 2014. 


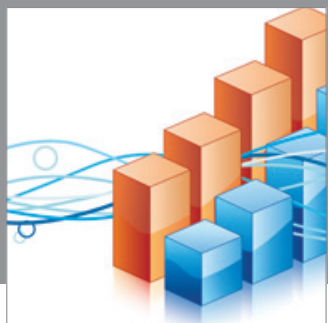

Advances in

Operations Research

mansans

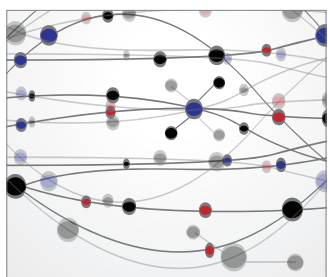

The Scientific World Journal
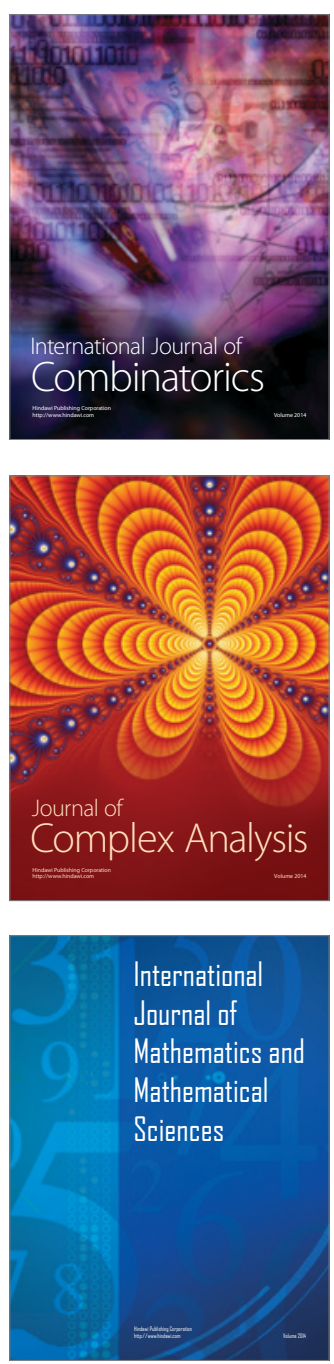
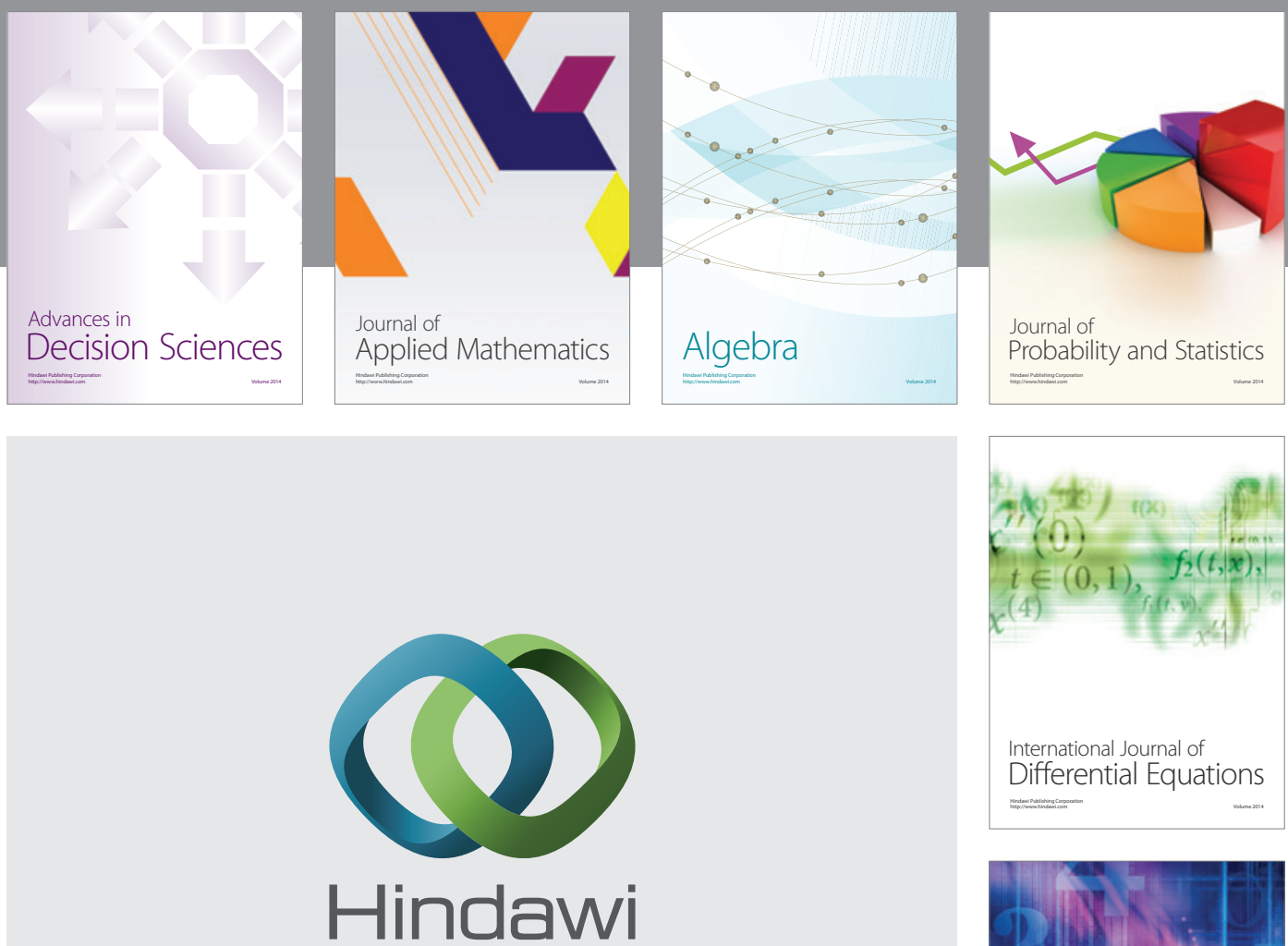

Submit your manuscripts at http://www.hindawi.com
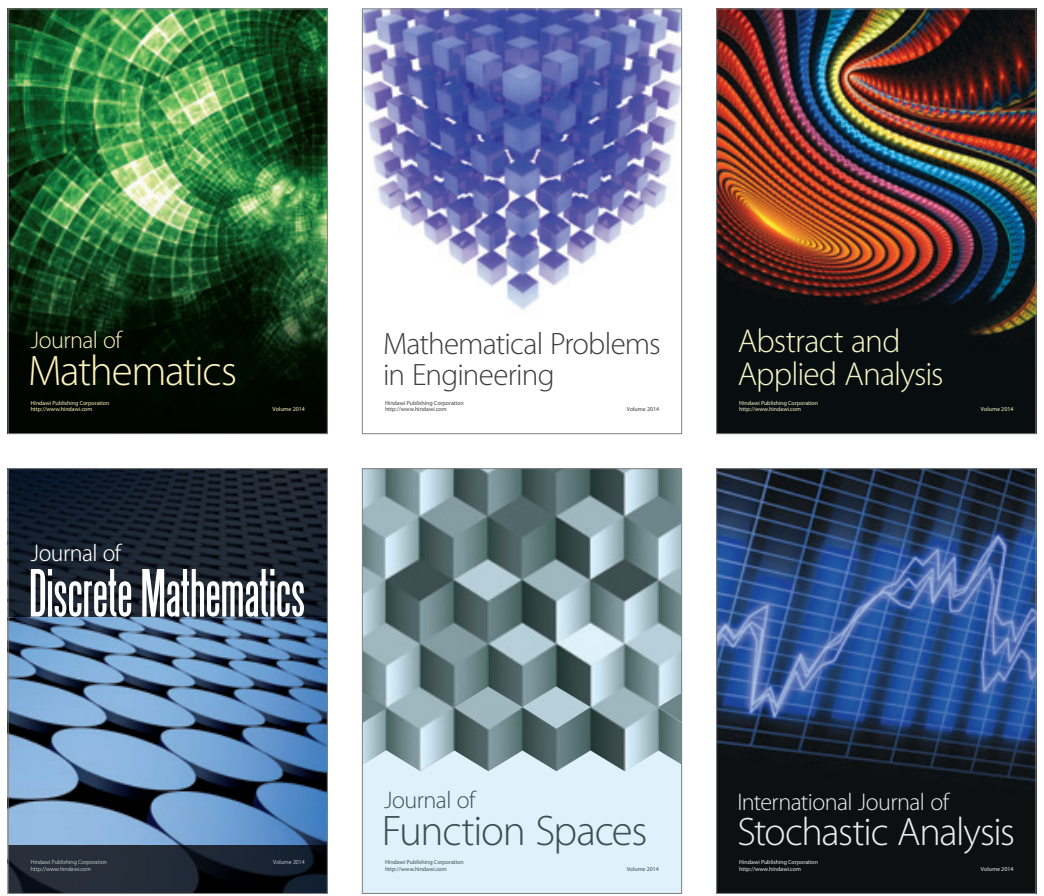

Journal of

Function Spaces

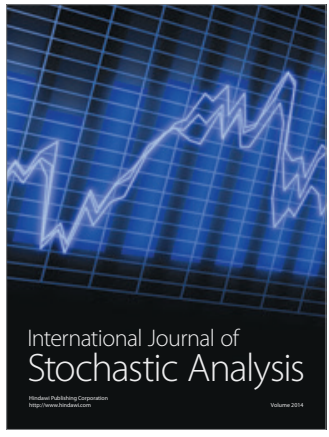

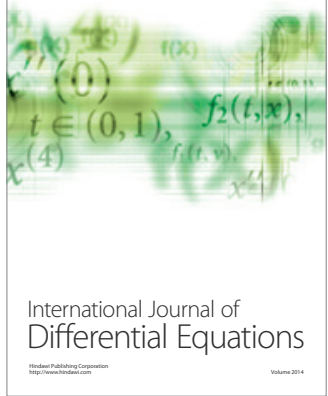
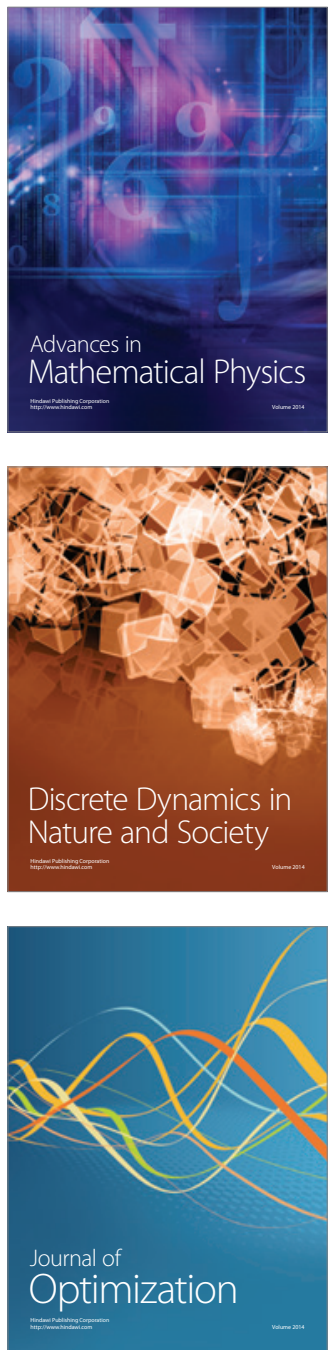